\title{
Characterisation of random Gaussian and non-Gaussian stress processes in terms of extreme responses
}

\author{
Bruno Colin ${ }^{\mathrm{a}}$ \\ Nexter Systems, 11 allées des Marronniers, 78022 Versailles Cedex, France
}

\begin{abstract}
In the field of military land vehicles, random vibration processes generated by all-terrain wheeled vehicles in motion are not classical stochastic processes with a stationary and Gaussian nature. Non-stationarity of processes induced by the variability of the vehicle speed does not form a major difficulty because the designer can have good control over the vehicle speed by characterising the histogram of instantaneous speed of the vehicle during an operational situation. Beyond this nonstationarity problem, the hard point clearly lies in the fact that the random processes are not Gaussian and are generated mainly by the non-linear behaviour of the undercarriage and the strong occurrence of shocks generated by roughness of the terrain. This non-Gaussian nature is expressed particularly by very high flattening levels that can affect the design of structures under extreme stresses conventionally acquired by spectral approaches, inherent to Gaussian processes and based essentially on spectral moments of stress processes. Due to these technical considerations, techniques for characterisation of random excitation processes generated by this type of carrier need to be changed, by proposing innovative characterisation methods based on time domain approaches as described in the body of the text rather than spectral domain approaches.
\end{abstract}

\section{Introduction}

In the context of design of structures under extreme stresses, it is usually accepted that stationary random excitation processes for these extreme stresses can be characterised by their acceleration PSD over a relevant frequency range, usually covering the LF [1 to $50 \mathrm{~Hz}$ ] and $\mathrm{MF}[50 \mathrm{~Hz}$ to $500 \mathrm{~Hz}$ ] ranges for the case of wheeled vehicles.

Methods of characterising extreme values generated by these random acceleration processes, based on conventional Gaussian signal assumptions, can then be used. This is the case particularly for characterisation techniques using the Response Spectrum (RS) method widely used by manufacturers in the Civilian and the Military fields for writing vibration design specifications. The Response Spectra (RS) technique consists of characterising the severity of the excitation process through a simple mechanical model composed of a linear system with a single degree of freedom $(1 \mathrm{df})$ in which the natural frequency $f_{0}$ is varied within a fairly wide range covering the natural frequencies of the structure to be designed and limited by the conventional effective masses criterion. In many industrial applications, this effective masses criterion consists of using all modes for which the sum of effective masses is $90 \%$ of the total mass of the structure to be designed [1]. At this stage, this is thus a generalisation of the Biot model historically used in calculation of the Shock Response Spectrum (SRS) of transient excitations and that is adapted to the case of random vibrations [2] referred to as an Extreme Response Spectrum (ERS). Thus, for a random vibration process usually evaluated on a limited portion $\mathrm{T}_{\text {ref }}$ of the given

\footnotetext{
a e-mail: b.colin@nexter-group.fr
}

total load duration $T_{t}$, its severity can be characterised by applying either a spectral domain (stochastic) approach or a time domain (deterministic) approach, each of which has disadvantages in the case of non-Gaussian random processes, as discussed below.

\section{Selected standard system}

When a vibrational excitation process is applied to the base of a mechanical system with one degree of freedom (mass - spring - damper), this standard system usually responds by generating a relative displacement between the mass and the base of the standard system (see Fig. 1). The maximum and minimum values of this relative displacement, denoted $z_{\text {sup }}$ and $z_{\text {inf }}$ respectively, then depend on the natural frequency $f_{0}$ of the standard and are proportional to the maximum and minimum stresses induced in the spring of this standard system. The designer imposes the proportionality constant K (Stress - Relative displacement) and it is kept constant regardless of the value of the natural frequency $f_{0}$.

Therefore we obtain:

$$
\sigma(t)=\mathrm{K} \cdot z(t) .
$$

Where $\sigma(t)$ : amplitude of the vibration stress.

$\mathrm{K}$ : proportionality constant between stress and relative displacement ( $\mathrm{K}$ is a constant if it is assumed that the structure to be designed is linear).

$z(t)$ : amplitude of the relative displacement.

Where:

$$
z(t)=y(t)-x(t)
$$

Where $y(t)$ : absolute displacement of the mass $m$. $x(t)$ : absolute displacement of the mobile base. 


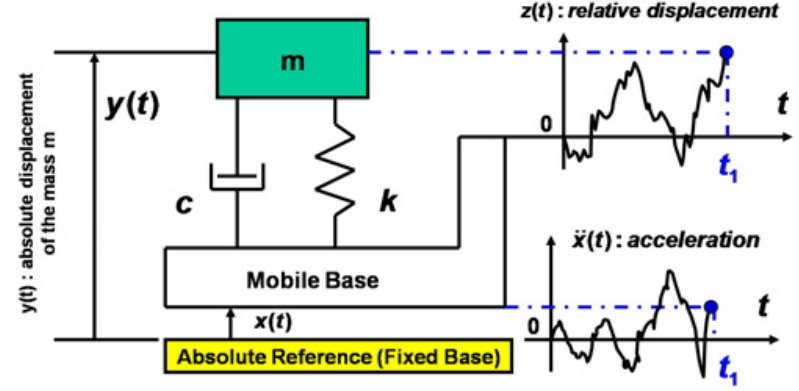

Figure 1. Standard system with 1 dof.

\subsection{Characteristics of the standard system}

The standard system with mass $\mathrm{m}$ (in $\mathrm{kg}$ ), stiffness $\mathrm{k}$ (in $\mathrm{N} / \mathrm{m}$ ) and viscous damping $\mathrm{c}$ (in N.s/m) is then characterised by its modal parameters, namely:

$$
\xi=\frac{\mathrm{c}}{2 \cdot \sqrt{\mathrm{k} \cdot \mathrm{m}}} \text { and } Q=1 / 2 . \xi \text { and } f_{0}=\frac{1}{2 \pi} \cdot \sqrt{\frac{\mathrm{k}}{\mathrm{m}}} \text {. }
$$

Where $\xi$ : critical or modal damping.

Q: modal overtension factor.

$f_{0}$ : natural or modal frequency $(\mathrm{Hz})$.

\subsection{Standard system transfer function}

The expression for the transfer function $H_{\ddot{x} z}(\omega=2 . \pi . f)$ of the standard system between excitation defined in terms of acceleration $\ddot{x}(t)$ and relative displacement $z(t)$ is as follows:

$$
\mathrm{H}_{\ddot{\mathrm{x} z}}(\omega)=\frac{\mathrm{Z}(\omega)}{\ddot{\mathrm{X}}(\omega)}=\left|\mathrm{H}_{\ddot{x} \mathrm{z}}(\omega)\right| . \mathrm{e}^{\mathrm{j} \cdot \angle \mathrm{H}_{\bar{x} z}(\omega)} .
$$

Where:

$$
\begin{gathered}
\mathrm{Z}(\omega)=\mathrm{TF}[\mathrm{z}(\mathrm{t})] \text { and } \ddot{\mathrm{X}}(\omega)=\mathrm{TF}[\ddot{\mathrm{x}}(\mathrm{t})] \\
\left|\mathrm{H}_{\ddot{\mathrm{x} z}}(\omega)\right| \text { modulus of the Transfer Function } \\
\angle \mathrm{H}_{\ddot{\mathrm{x} z}}(\omega) \text { phase of the Transfer Function } \\
\mathrm{TF}[.] \text { Fourier Transform operator }
\end{gathered}
$$

Furthermore, all that is required to calculate the ERS at this stage is the modulus of the transfer function, if the spectral method described in Sect. 3.1 according to [3] is used. In non-dimensional form, we can write:

$$
\left|\mathrm{H}_{\ddot{\mathrm{x} Z}}(\omega)\right|=\frac{1}{\omega_{0}^{2}} \cdot \frac{1}{\sqrt{\left(1-\mathrm{h}^{2}\right)^{2}+4 \cdot \xi^{2} \cdot \mathrm{h}^{2}}}
$$

where $\mathrm{h}=\frac{\omega}{\omega_{0}}=\frac{f}{f_{0}}, \omega_{0}=\sqrt{\frac{\mathrm{k}}{\mathrm{m}}}=2 . \pi . f_{0}$ et $\mathrm{Q}=1 / 2 . \xi$.

\section{ERS calculation approaches}

As mentioned in the introduction, there are two possible approaches for calculating the ERS of a stationary random excitation process, depending on whether we want to:

- consider a stochastic approach to extrapolate maximum values of the random stress process over the total load duration $T_{t}$, the values usually being estimated over a limited reference duration $\mathrm{T}_{\text {ref }}\left(\mathrm{T}_{\text {ref }}<\mathrm{T}_{\mathrm{t}}\right)$ for reasons of calculation or measurement time. This point is the strong point of stochastic methods historically developed around spectral techniques,

- or consider a deterministic approach which calculates only maximum values of the random stress process over duration $\mathrm{T}_{\text {ref }}$, without being able to extrapolate them satisfactorily over the total load duration $T_{t}$ considered. Consequently, it is clear that this time domain calculation method is not conservative and can lead to serious mechanical underdesign for studied structures. This point is particularly true if the duration $\mathrm{T}_{\text {ref }}$ is short compared with the total duration $\mathrm{T}_{\mathrm{t}}$ of the load considered.

Unlike the case for spectral methods, time domain approaches can require prohibitive calculation times in addition to the identified risk of underdesign. The advantage of spectral methods is the fact that the ERS calculation is analytic, although there is the concept of an associated exceedance risk $\alpha$ that has to be specified. For example, considering the simplified case of stationary Gaussian and random processes, it can be demonstrated that this spectral ERS concept is implicitly associated with an exceedance risk of $63.2 \%$. This is not a very reassuring point for designers who are responsible for complex structures forming part of a high performance functional system. This is why the classical spectral ERS model was generalised by spectral XRS approaches proposed by the author in 2007 [4].

\subsection{Calculation of the spectral ERS}

There are different approaches for calculating the spectral ERS; the approach proposed herein is based on the following elements:

- the excitation process is stationary, ergodic, Gaussian and has zero mean (therefore all spectral moments are constant and can be calculated from the PSD of the relative displacement of the standard system),

- since the selected standard system is linear, the relative displacement $\mathrm{z}(\mathrm{t})$ of the model is itself stationary, ergodic, Gaussian and has zero mean. Furthermore, the standard system has an overtension factor $\mathrm{Q} \geq 3$, consequently the random stress process is narrow band in which the local maxima are characterised by a Rayleigh law,

- work done by S.O Rice [5] on narrow band Gaussian processes has shown that the average ratio of local maxima $E\left[\overline{N_{\max }}(\xi)\right]$ as defined in the Peak-Valley extraction method, is equal to the average rate at which the random stress process defined in terms of relative displacement $\mathrm{z}(\mathrm{t})$ passes through zero with a positive gradient $E\left[\overline{N_{0}^{+}}(\xi)\right]$. Consequently, the conservative spectral approach often leads to the assumption that the stress process in terms of relative displacement is narrow band centred on its natural frequency $f_{0}$. Therefore, we can write:

$$
E\left[\overline{N_{\max }}(\xi)\right]=E\left[\overline{N_{0}^{+}}(\xi)\right]=f_{0} .
$$


From these technical considerations, we can define the analytic formulation of the probability density law of local maxima $f_{Z_{\max }}(z)$ of the random process $\mathrm{z}(\mathrm{t})$ that is a Rayleigh law with parameter $z_{e f f}$, expressed as follows [6]:

$$
z_{e f f}=\left[\int_{f=0}^{+\infty} D S P_{z z}(f) \cdot d f\right]^{1 / 2} .
$$

In which:

$$
D S P_{z z}(f)=\left|H_{\ddot{x} z}(\omega)\right|^{2} . D S P_{\ddot{x} \ddot{x}}(f) .
$$

Where: $D S P_{z z}(f)$ : PSD of the random stress process $\mathrm{z}(\mathrm{t})$ $D S P_{\ddot{x} \ddot{x}}(f)$ : PSD of the random excitation process $\ddot{x}(t)$.

Finally, the analytic expression of the distribution function of local maxima $F_{Z_{\max }}(z)$ of the stress process $\mathrm{z}(\mathrm{t})$ from which the spectral ERS calculation is determined has to be defined. By definition, this distribution function is obtained by integrating the distribution law of local maxima defined above, and the result obtained is:

$$
\begin{gathered}
F_{Z_{\max }}(z)=\operatorname{Prob}\left[Z_{\max }(\xi) \leq z\right]=1-\exp \left[-\frac{z^{2}}{2 \cdot z_{\text {eff }}^{2}}\right] \\
\text { where } z \in[0,+\infty[.
\end{gathered}
$$

Thus by definition, the maximum value $z_{\text {sup }}$ associated with the random process $\mathrm{z}(\mathrm{t})$ is equal to the maximum value that occurs on average only once during the total load duration $\mathrm{T}_{\mathrm{t}}$ of the excitation process considered, so we can write:

$$
F_{Z_{\max }}\left(z_{\text {sup }}\right)=1-\frac{1}{E\left[\overline{N_{\max }}(\xi)\right] \cdot T_{t}}=1-\frac{1}{f_{0} \cdot T_{t}} .
$$

Hence the expression of the spectral ERS (narrow band) $S R E_{s p}^{B E}\left(f_{0}\right)$, namely:

$$
\begin{gathered}
\qquad S R E_{s p}^{B E}\left(f_{0}\right)=\omega_{0}^{2} \cdot z_{\text {sup }}\left(f_{0}\right) \\
\text { where } z_{\text {sup }}\left(f_{0}\right)=z_{\text {eff }}\left(f_{0}\right) \cdot \sqrt{2 \cdot \ln \left(f_{0} \cdot T_{t}\right)} \\
\text { and } z_{\text {eff }}^{2}\left(f_{0}\right)=\int_{f=0}^{+\infty} D S P_{z z}(f) \cdot d f .
\end{gathered}
$$

Therefore as described above, the result obtained is a simple analytic expression for the spectral ERS (in this case narrow band) with the advantage that maximum stresses can be extrapolated over the total duration $T_{t}$ of the excitation process considered, while the excitation process is usually estimated over a reference period $\mathrm{T}_{\text {ref }}$ shorter than $T_{t}$. This simple expression of the narrow band spectral ERS can be generalised to the case of wide band processes [7] that will be denoted $\operatorname{SRE} E_{s p}^{B L}\left(f_{0}\right)$, by analogy with $S R E_{s p}^{B E}\left(f_{0}\right)$. Nevertheless, the disadvantage of these spectral methods is that they are dependent on a restrictive Gaussian assumption.

\subsection{Calculation of the time domain ERS (with deterministic nature)}

At this stage, the calculation of the time domain ERS consists of evaluating the maximum value of a sample $\mathrm{z}(\mathrm{t})$ of the stress process, based exclusively on a time sample $\ddot{x}(t)$ of the excitation process defined over its reference duration $T_{\text {ref }}$ shorter than the total duration $T_{t}$ of the excitation process considered $\left(\mathrm{T}_{\text {ref }}<\mathrm{T}_{\mathrm{t}}\right)$. This is done by calculating a time sample of the stress process $\mathrm{z}(\mathrm{t})$, defined in terms of relative displacement by time domain methods. There are different approaches for calculating the deterministic time domain ERS $S R E_{t p}^{D}\left(f_{0}\right)$, namely:

- The digital convolution method based on the Duhamel integral that is a method well described in the literature. However, its principal disadvantage is in the fact that it is expensive to compute due to its non-recursive nature, leading to economically inefficient calculation times [8].

- Recursive methods or recursive digital filters based on the technique of state transition or $\mathrm{Z}$ transformation matrices well known to automatic control experts [9]. The saving in calculation time achieved with these recursive methods in comparison with digital convolution techniques makes them high performance and therefore inevitable, once reference times $\mathrm{T}_{\text {ref }}$ are longer than a few hundred minutes.

Consequently, this deterministic ERS calculation method is applicable to all stationary vibration samples with no restrictions. Therefore, this is an alternative calculation method for the case of non-Gaussian stationary random processes, but with the disadvantage of not being able to extrapolate maximum $\mathrm{z}_{\text {sup }}$ and minimum $\mathrm{z}_{\text {inf }}$ values of $\mathrm{z}(\mathrm{t})$ for a duration longer than $\mathrm{T}_{\text {ref }}$ and therefore equal to the total load duration $T_{t}$ of the excitation process considered. For information, an analytic expression of the digital convolution method that is expressed from the pulse function of the standard system is given below:

$$
\mathrm{h}_{\ddot{\mathrm{xz}} \mathrm{z}}(t)=\frac{-1}{\omega_{\mathrm{d}}} \cdot \mathrm{e}^{-\xi \cdot \omega_{0} \cdot \mathrm{t}} \cdot \sin \left[\omega_{\mathrm{d}} \cdot \mathrm{t}\right] \quad \text { for } t \geq 0
$$

where $\omega_{\mathrm{d}}=\omega_{0} \cdot \sqrt{1-\xi^{2}}$ and $\xi \leq 1, \quad$ namely $\mathrm{Q} \geq 0,5$.

The time sample $\mathrm{z}(\mathrm{t})$ of the stress process in terms of relative displacement is then obtained by the Duhamel integral over the reference duration $\mathrm{T}_{\text {ref }}$ of the excitation sample $\ddot{x}(t)$ to be characterised. Therefore, this expression is written:

$$
\mathrm{z}(\mathrm{t})=\left\{\begin{array}{l}
\int_{\tau=0}^{t} \mathrm{~h}_{\ddot{\mathrm{X} z}}(t-\tau) \cdot \ddot{\mathrm{x}}(\tau) \cdot \mathrm{d} \tau \\
\frac{-1}{\omega_{\mathrm{d}}} \cdot \int_{\tau=0}^{t} \ddot{\mathrm{x}}(\tau) \cdot \mathrm{e}^{-\xi \cdot \omega_{0} \cdot(t-\tau)} \cdot \sin \left[\omega_{\mathrm{d}} \cdot(t-\tau)\right] \cdot \mathrm{d} \tau
\end{array}\right.
$$

$$
\text { for } t \in\left[0, t_{\text {réf }}\right] \text {. }
$$

Where:

$$
\omega_{\mathrm{d}}=\omega_{0} \cdot \sqrt{1-\xi^{2}} \text { and } \xi \leq 1, \quad \text { namely } \mathrm{Q} \geq 0,5 .
$$

Now that the shape of $z(t)$ is known for $t$ varying from 0 to $\mathrm{T}_{\text {ref }}$, the expression of the time domain ERS with a deterministic nature can be evaluated based on the 
following expression:

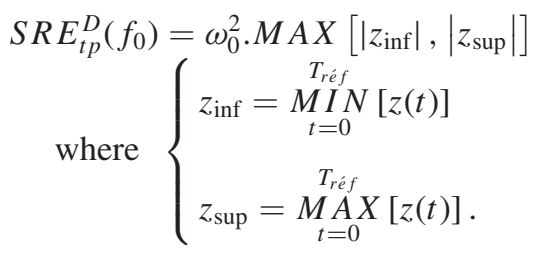

This technique for calculating the time domain ERS with a deterministic nature is very easy to develop digitally [10] because it is similar to the SRS technique, but is very often unsuitable for a secure structure design context which necessarily requires that the total duration $T_{t}$ of stochastic stress processes should be taken into account. Therefore this necessarily involves the use of probabilistic and no longer deterministic methods capable of extrapolating extreme values of processes over the duration $T_{t}$, with an exceedance risk $\alpha$ that can be calculated and is therefore controlled.

\section{Security of structures and control over the design risk}

In the future, we should consider the largest extreme values generated by these processes over the duration $T_{t}$ to secure the design of mechanical structures under stationary random processes with the load duration $T_{t}$, by imposing an exceedance risk $\alpha$ that is fixed as a function of the criticality of the structure to be designed.

In practice, the three risk levels usually used in the mechanical design for land military vehicles are:

$-\alpha=10 \%$ : risk associated with the development of "routine" mechanical equipment and/or structures that do not form part of a functional system for which performance is important for the final client. This is particularly the case for devices associated with secondary functions of the system,

$-\alpha=1 \%$ : risk associated with the development of "standard" mechanical equipment and/or structures forming part of a functional system (sensitive) for which performance is important for the final client, but for which the client requirement level remains moderate. This is the case particularly for devices associated with the main functions of the System, for which functional performance levels specified by the final client can be achieved with no risk, with regard to vibration stresses assumed in the development,

$-\alpha=0,1 \%$ : risk associated with the development of "secure" mechanical equipment and/or structures forming part of a functional system for which performance is important for the final client, but for which the client requirement level is high. This is the case particularly for devices associated with the principal functions of the System, for which high functional performance levels specified by the final client cannot be achieved without risk unless the loads assumed in the development are well controlled.

Therefore, from these technical considerations, during the period from 2005 to $2010[4,7]$ it became clear that major changes needed to be made to Response Spectrum
(RS) techniques to get a better understanding of these extreme value exceedance risks, in the case of excitation processes generated by Land military vehicles. This work started in the context of Gaussian random processes so that the concept of a spectral XRS with an imposed exceedance risk $\alpha$ could be implemented to replace the case of the spectral ERS (wide band or narrow band) implicitly associated with a risk of $63.2 \%$. This spectral XRS concept was used successfully for specifications in a mechanical environment to make favourable comparisons between deterministic processes (Sinusoidal Shocks and Vibrations) and stochastic type phenomena (Random vibrations), in terms of extreme stresses [11].

Therefore Sect. 5 below summarises the main results of this spectral XRS concept that is dependent on the case of Gaussian processes. This will usefully be extended by innovative approaches so that time domain XRS concepts can be proposed capable of taking account of the nonGaussian nature of random processes generated by Land vehicles.

\section{XRS calculation approaches}

As already mentioned, it is now essential to be able to estimate maximum values of random stress processes over the total duration $\mathrm{T}_{t}$ of the excitation process, regardless of the statistical nature of the excitation process (Gaussian or non-Gaussian). Therefore this leads to no longer favouring the use of deterministic time domain ERS techniques that appeared to be widespread by designers, if probabilistic methods adapted to the required technical challenge are not available. The objective in the future will be to evaluate the highest stress threshold over the total excitation duration $\mathrm{T}_{t}$, with an imposed exceedance risk $\alpha$ rather than a fixed value as is the case for the spectral ERS (narrow band or wide band).

These Response Spectra with imposed exceedance risk $\alpha$ have been denoted XRS to differentiate them from the term ERS and they now cover the case of Gaussian and/or non-Gaussian stationary random processes, as presented below.

\subsection{Calculation of the spectral XRS}

There are different approaches to calculating the spectral $\mathrm{XRS}$, the approach proposed below is based on the following elements:

- the excitation process is stationary, ergodic, Gaussian and has zero mean (therefore all spectral moments are constant and can be calculated from the PSD of the relative displacement of the standard system),

- since the selected standard system is linear, the relative displacement $z(t)$ of the model is itself stationary, ergodic, Gaussian and has zero mean. Furthermore, since the standard system has an overtension factor $\mathrm{Q} \geq 3$, the random stress process is narrow band, with global maxima that occur over the total duration $\mathrm{T}_{t}$ characterised asymptotically by a Poisson or Gumbel law. These two statistical approaches are very similar for long load cycles $\left(n=f_{0} . T_{t}\right)$. 
Based on Poisson's asymptotic law, it is easy to demonstrate that the expression for the distribution function for the largest of the $\mathrm{n}$ maxima $\left(Y_{n}\right)$ of the stress process $\mathrm{z}(\mathrm{t})$ is:

$$
\begin{aligned}
& F_{Y_{n}}^{P}(\eta) \underset{n \text { grand }}{\longrightarrow} \exp \left[-n \cdot \exp \left[-\frac{\eta^{2}}{2}\right]\right] \\
& \text { where } n=f_{0} \cdot T_{t} \quad \text { and } \eta=\frac{z}{z_{\text {eff }}}>0 .
\end{aligned}
$$

Hence the expression for the spectral XRS (according to meaning in the Poisson model), namely:

$$
\begin{aligned}
& \operatorname{SRX} X_{s p}^{P}\left(f_{0}\right)=\omega_{0}^{2} \cdot z_{\alpha}^{P}\left(f_{0}\right) \text { where } F_{Y_{n}}^{P}\left(\eta_{\alpha}^{P}=\frac{z_{\alpha}^{P}}{z_{e f f}}\right)=1-\alpha \\
& \Rightarrow z_{\alpha}^{P}\left(f_{0}\right)=z_{e f f} \cdot \sqrt{2 \cdot\left[\ln \left(f_{0} \cdot T_{t}\right)-\ln [-\ln (1-\alpha)]\right]} \\
& \text { Hence: } z_{\alpha}^{P}\left(f_{0}\right)=z_{e f f} \cdot \sqrt{2 \cdot \ln \left(f_{0} \cdot T_{t}\right)} \\
& \quad \sqrt{1-\frac{\ln [-\ln (1-\alpha)]}{\ln \left(f_{0} \cdot T_{t}\right)}} .
\end{aligned}
$$

Concerning Gumbel's asymptotic law, it is easy to demonstrate that expression for the distribution function of the largest of the $\mathrm{n}$ maxima $\left(Y_{n}\right)$ of the stress process $z(t)$ is:

$$
\left\{\begin{array}{l}
F_{Y_{n}}^{G}(z) \underset{n \text { grand }}{\longrightarrow} \exp \left[-\exp \left[-\alpha_{n} \cdot\left(z-u_{n}\right)\right]\right] \\
\text { for } y \in]-\infty,+\infty[
\end{array}\right.
$$

Where:

$$
\begin{aligned}
& n=f_{0} \cdot T_{t}, \quad z_{\text {eff }}^{2}=\int_{f=0}^{+\infty} \operatorname{DSP}_{z z}(f) d f \\
& \text { and } \quad u_{n}=z_{\text {eff }} \cdot \sqrt{2 \cdot \ln (n)}, \quad \alpha_{n}=u_{n} / z_{\text {eff }}^{2} .
\end{aligned}
$$

Hence the expression for the spectral XRS (according to meaning in the Gumbel model), namely:

$$
\begin{gathered}
S R X_{s p}^{G}\left(f_{0}\right)=\omega_{0}^{2} \cdot z_{\alpha}^{G}\left(f_{0}\right) \text { where } F_{Y_{n}}^{G}\left(z_{\alpha}^{G}\right)=1-\alpha \\
\Rightarrow z_{\alpha}^{G}\left(f_{0}\right)=u_{n} \cdot\left[1-\frac{\ln [-\ln (1-\alpha)]}{u_{n} \cdot \alpha_{n}}\right] \\
\text { Hence: } z_{\alpha}^{G}\left(f_{0}\right)=z_{e f f} \cdot \sqrt{2 \cdot \ln \left(f_{0} \cdot T_{t}\right)} . \\
{\left[1-\frac{\ln [-\ln (1-\alpha)]}{2 \cdot \ln \left(f_{0} \cdot T_{t}\right)}\right] .}
\end{gathered}
$$

Since the expressions of the narrow band spectral XRS derived from the two asymptotic models (Poisson and Gumbel) are identical [4] if the average number of cycles $\mathrm{n}$ is more than $1 \mathrm{E}+04$, in practice the Gumbel model will be used because it is a concept that can be easily generalised to the case of wide band Gaussian and nonGaussian processes as presented below.

\subsection{Calculation of the time domain XRS}

It is no longer possible to use spectral approaches to evaluate the XRS of a stationary Gaussian process with duration $\mathrm{T}_{t}$ characterised by a single time domain stress sample $\mathrm{z}(\mathrm{t})$ with limited duration $\mathrm{T}_{\text {ref }}\left(\mathrm{T}_{\text {ref }}<\mathrm{T}_{\mathrm{t}}\right)$. Although these non-Gaussian processes can always be characterised in frequency by their PSD, the probabilistic structure of their local maxima is only accessible by analysing their first statistical moments evaluated over the period $\left[0, \mathrm{~T}_{\mathrm{ref}}\right]$. From now on, the time domain approach set up in the case of a random non-Gaussian process will consist of analysing their maximum local values of the stress process in blocks with duration $\mathrm{T}_{\mathrm{b}}$ (the author calls this the disjoint block method).

The number of analysis blocks $\mathrm{N}$ is then equal to the ratio $\left(\mathrm{T}_{r e ́ f} / \mathrm{T}_{b}\right)$ and the maximum value $Z_{\max }(i)$ of each block $i=1$ to $\mathrm{N}$ is estimated. The sample of $\mathrm{N}$ maximum values $\left\{\mathrm{Z}_{\max }(i), i=1 \grave{a} N\right\}$ thus evaluated over the duration $\mathrm{T}_{\text {ref }}$, is such that the first statistical moments of the largest maxima $Z_{\max }$ of $\mathrm{z}(\mathrm{t})$ arriving over the duration $\mathrm{T}_{\mathrm{b}}$ can be characterised. The first two moments of $Z_{\max }$ can then be easily calculated and their expressions are the following relations in terms of the average $\hat{\bar{Z}}_{\max }$ and variance $\hat{\sigma}_{Z_{\max }}^{2}$ :

$$
\hat{\bar{Z}}_{\max }=\frac{1}{\mathrm{~N}} \cdot \sum_{\mathrm{i}=1}^{\mathrm{N}} \mathrm{Z}_{\max }(\mathrm{i}) \quad \text { where } \mathrm{N}=\mathrm{T}_{\text {réf }} / \mathrm{T}_{\mathrm{b}} .
$$

And,

$$
\left\{\begin{array}{l}
\hat{\sigma}_{\mathrm{Z}_{\max }}^{2}=\frac{1}{\mathrm{~N}-1} \cdot \sum_{\mathrm{i}=1}^{\mathrm{N}}\left[\mathrm{Z}_{\max }(\mathrm{i})-\hat{\mathrm{Z}}_{\max }\right]^{2} \\
\text { where } \mathrm{N}=\mathrm{T}_{\text {réf }} / \mathrm{T}_{\mathrm{b}} .
\end{array}\right.
$$

Thus, for a total given load time $T_{t}$, associated with the random excitation process and characterised by its acceleration $\left\{\ddot{x}(t), t \in\left[0, T_{t}\right]\right\}$, it is now possible to define the distribution function of the largest maxima of $\mathrm{z}(\mathrm{t})$, denoted $Y_{M}$ over this total load time $\mathrm{T}_{\mathrm{t}}=\mathrm{M}_{\mathrm{T}} \mathrm{T}_{\mathrm{b}}$ where $M$ represents the extrapolation factor in duration of the process. This can be done using the distribution function of $Z_{\max }$, for which the first two statistical moments are known by (22) and (23).

Continuing the work done for the spectral XRS, it seems logical to use the Gumbel asymptotic model once again to describe the distribution function of $Y_{M}$. To achieve this, a sufficiently short block duration $T_{b}$ has to be used so that $\mathrm{M}$ can be assumed to be high, and distribution laws $f_{Z_{\max }}(z)$ for $Z_{\max }$ belonging to the Gumbel attraction domain also have to be used. Therefore, we can write:

$$
\begin{gathered}
F_{Y_{M}}(y) \underset{M \text { grand }}{\longrightarrow} \exp \left[-\exp \left[-\alpha_{M} \cdot\left(y-u_{M}\right)\right]\right] \\
\text { where } \left.M=T_{t} / T_{b}, \quad y \in\right]-\infty,+\infty[ \\
f_{Z_{\max }}(z)=\frac{d\left[F_{Z_{\max }}(z)\right]}{d z} \\
\text { and } u_{M}=F_{Z_{\max }}^{-1}\left(1-\frac{1}{M}\right), \alpha_{M}=M \cdot f_{Z_{\max }}\left(u_{M}\right) .
\end{gathered}
$$

The author has developed two types of time domain XRS models from these technical considerations for the case of non-Gaussian processes generated by wheeled Land vehicles. These are the time domain XRS models of the 
Log-Normal or Weibull type, depending on whether the distribution law of local maxima $Z_{\max }$ is of the LogNormal or Weibull type. The fundamental results of these two approaches are given below.

\subsubsection{Log-Normal approach}

Assuming that the distribution law of local maxima of the stress process $Z_{\max }$ is of the Log-Normal type and has parameters $(\mu, \sigma)$, analytic expressions for these two parameters can be evaluated based principally on the statistical moments theory. These expressions then depend on $\hat{\bar{Z}}_{\max }$ and $\hat{\sigma}_{Z_{\max }}$ and the empirical variation coefficient of $Z_{\max }$, for which the expression is:

$$
C V\left[Z_{\max }\right]=\hat{\sigma}_{Z_{\max }} / \hat{\bar{Z}}_{\max } .
$$

We then obtain:

$$
\begin{aligned}
& f_{Z_{\max }}^{L N}(z)=L N(\mu, \sigma)=\frac{1}{z \cdot \sigma \cdot \sqrt{2 \cdot \pi}} \cdot \exp \left[-\frac{1}{2} \cdot\left(\frac{\ln z-\mu}{\sigma}\right)\right] \\
& \mu=\ln \left[\hat{\bar{Z}}_{\max }\right]-\frac{\sigma^{2}}{2}, \quad \sigma^{2}=\ln \left[1+C V\left[Z_{\max }\right]\right], \quad z>0 .
\end{aligned}
$$

Since the distribution function of $Z_{\max }$ is now known analytically, the analytic expression of its distribution function can also be defined so that we can write:

$$
\begin{gathered}
F_{Z_{\max }}^{L N}(z)=\frac{1}{2} \cdot\left[1+\operatorname{erf}\left(\frac{\ln z-\mu}{\sigma \cdot \sqrt{2}}\right)\right] \text { for } z \in[0,+\infty[ \\
\text { where } \operatorname{erf}(x)=\frac{2}{\sqrt{\pi}} \cdot \int_{t=0}^{x} \exp \left(-t^{2}\right) d t .
\end{gathered}
$$

As mentioned above, assuming that the largest maxima $Y_{M}$ of $\mathrm{z}(\mathrm{t})$ over the total duration $\mathrm{T}_{t}$ is described by a Gumbel asymptotic law, the expression of the time domain XRS denoted $S R X_{t p}^{L N}\left(f_{0}\right)$ can be defined for this Log-Normal approach, namely:

$S R X_{t p}^{L N}\left(f_{0}\right)=\omega_{0}^{2} \cdot y_{\alpha}^{L N}\left(f_{0}\right) \quad$ where $\quad F_{Y_{M}}\left(y_{\alpha}^{L N}\right)=1-\alpha$

$$
\begin{gathered}
\text { where } y_{\alpha}^{L N}=u_{M} \cdot\left[1-\frac{\ln [-\ln (1-\alpha)]}{u_{M} \cdot \alpha_{M}}\right] \\
\text { and }\left\{\begin{array}{l}
u_{M}=\exp \left[\mu+\sigma \cdot \sqrt{2} \cdot \operatorname{erf}^{-1}\left(\frac{M-2}{M}\right)\right] \\
\alpha_{M}=\frac{M}{u_{M} \cdot \sigma \cdot \sqrt{2 \cdot \pi}} \cdot \exp \left[-\left[\operatorname{erf}^{-1}\left(\frac{M-2}{M}\right)\right]^{2}\right] .
\end{array}\right.
\end{gathered}
$$

By definition, since the time domain ERS $S R E_{t p}^{L N}\left(f_{0}\right)$ is associated with an exceedance risk of $63.2 \%$, we can write the following for this Log-Normal approach:

$$
\frac{S R X_{t p}^{L N}\left(f_{0}\right)}{S R E_{t p}^{L N}\left(f_{0}\right)}=1-\left[1-\frac{\ln [-\ln (1-\alpha)]}{u_{M} \cdot \alpha_{M}}\right]
$$

where $\quad u_{M} \cdot \alpha_{M}=\frac{M}{\sigma \cdot \sqrt{2 \cdot \pi}} \cdot \exp \left[-\left[\operatorname{erf}^{-1}\left(\frac{M-2}{M}\right)\right]^{2}\right]$.

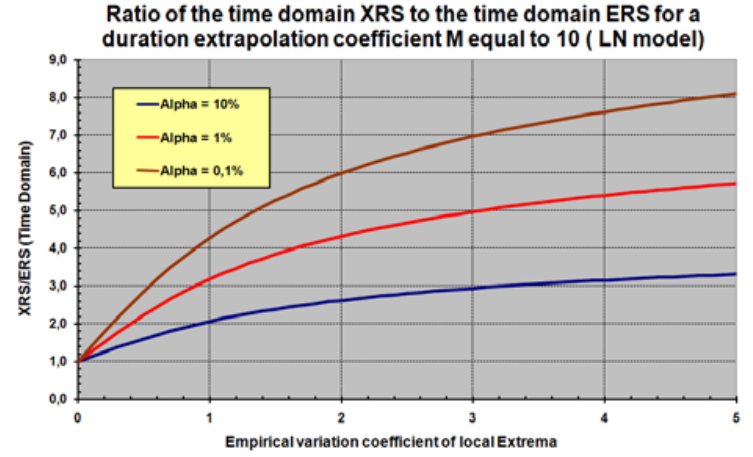

Figure 2. $\mathrm{XRS}_{\mathrm{tp}} / \mathrm{ERS}_{\mathrm{tp}}(\mathrm{LN}$ model) for $\mathrm{M}=10$.

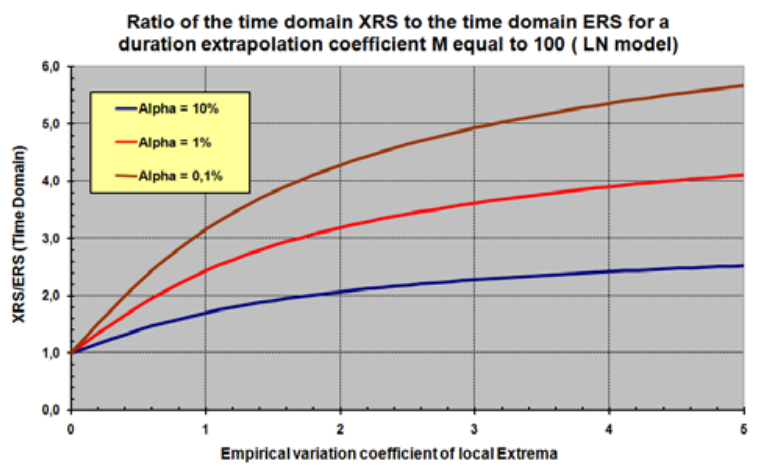

Figure 3. $\mathrm{XRS}_{\mathrm{tp}} / \mathrm{ERS}_{\mathrm{tp}}(\mathrm{LN}$ model $)$ for $\mathrm{M}=100$.

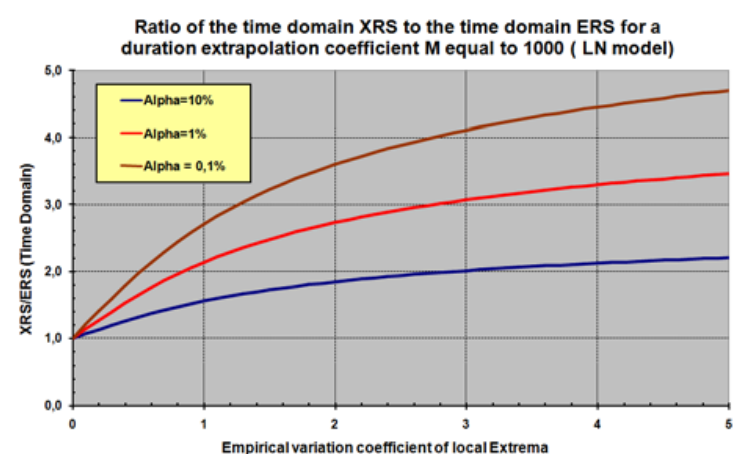

Figure 4. $\mathrm{XRS}_{\mathrm{tp}} / \mathrm{ERS}_{\mathrm{tp}}(\mathrm{LN}$ model) for $\mathrm{M}=1000$.

Therefore, it can be seen that the ratio of the time domain $\mathrm{XRS}$ to the time domain ERS is a function of $\alpha, \mathrm{M}$ and $\mathrm{CV}\left[\mathrm{Z}_{\max }\right]$ that can be shown graphically as follows for a duration extrapolation coefficient $\mathrm{M}$ equal to 10,100 or 1000 (plots shown in Figs. 2, 3 and 4 respectively).

\subsubsection{Weibull approach}

Assuming that the distribution law of local maxima of the stress process $Z_{\max }$ is of the Weibull type and has parameters $(\eta, \beta)$, we can write:

$$
f_{Z_{\max }}^{W B}(z)=W B(\eta, \beta)=\frac{\beta}{\eta} \cdot\left(\frac{z}{\eta}\right)^{\beta-1} \cdot \exp \left[-\left(\frac{z}{\eta}\right)^{\beta}\right]
$$
where $z \in]-\infty,+\infty[$. 
Hence the expressions for the two parameters of the Weibull law considered:

$$
\begin{aligned}
\eta=\frac{\hat{\bar{Z}}_{\max }}{\Gamma\left(1+\frac{1}{\beta}\right)}, & \text { and } \beta / g\left(\beta, C V\left[Z_{\max }\right]\right)=0 \\
g\left(\beta, C V\left[Z_{\max }\right]\right)= & {\left[\frac{\Gamma\left(1+\frac{2}{\beta}\right)-\Gamma^{2}\left(1+\frac{1}{\beta}\right)}{\Gamma^{2}\left(1+\frac{1}{\beta}\right)}\right] } \\
& -\left(C V\left[Z_{\max }\right]\right)^{2}
\end{aligned}
$$

Where $\Gamma(x)=\int_{u=0}^{+\infty} \exp (-u) \cdot u^{x-1} \cdot d u$ (Gamma function).

Since the distribution function of $Z_{\max }$ is now known analytically, the analytic expression for its distribution function can also be defined so that we can write:

$$
F_{Z_{\max }}^{W B}(z)=1-\exp \left[-\left(\frac{z}{\eta}\right)^{\beta}\right] \quad \text { for } \quad z \in[0,+\infty[.
$$

As mentioned above, assuming that the largest maxima $Y_{M}$ of $\mathrm{z}(\mathrm{t})$ over the total duration $\mathrm{T}_{\mathrm{t}}$ is described by a Gumbel asymptotic law, with this Weibull approach the expression of the time domain XRS denoted $S R X_{t p}^{W B}\left(f_{0}\right)$ can be defined, namely:

$$
\begin{gathered}
S R X_{t p}^{W B}\left(f_{0}\right)=\omega_{0}^{2} \cdot y_{\alpha}^{W B}\left(f_{0}\right) \quad \text { where } F_{Y_{M}}\left(y_{\alpha}^{W B}\right)=1-\alpha \\
\text { where } y_{\alpha}^{W B}=u_{M} \cdot\left[1-\frac{\ln [-\ln (1-\alpha)}{u_{M} \cdot \alpha_{M}}\right] \\
\text { and }\left\{\begin{array}{l}
u_{M}=\eta \cdot[\ln (M)]^{1 / \beta} \\
\alpha_{M}=\frac{\beta}{\eta} \cdot[\ln (M)]^{\frac{\beta-1}{\beta}} .
\end{array}\right.
\end{gathered}
$$

By definition, since the time domain $\operatorname{ERS} S R E_{t p}^{W B}\left(f_{0}\right)$ is associated with an exceedance risk of $63.2 \%$, it is found that for this Weibull approach we can write:

$$
\begin{gathered}
\frac{S R X_{t p}^{W B}\left(f_{0}\right)}{S R E_{t p}^{W B}\left(f_{0}\right)}=1-\left[1-\frac{\ln [-\ln (1-\alpha)]}{u_{M} \cdot \alpha_{M}}\right] \\
\text { where } u_{M} \cdot \alpha_{M}=\beta \cdot[\ln (M)] .
\end{gathered}
$$

Therefore, it can be observed that the ratio of the time domain XRS to the time domain ERS is once again a function of $\alpha, \mathrm{M}$ and $\mathrm{CV}\left[\mathrm{Z}_{\max }\right]$ that can be shown graphically as follows for a duration extrapolation coefficient $\mathrm{M}$ equal to 10,100 or 1000 (plots shown in Figs. 5, 6 and 7 respectively).

\subsubsection{Comments}

Based on results of Sects. 5.2.1 and 5.2.2, it can be observed that the behaviours of the Log-Normal

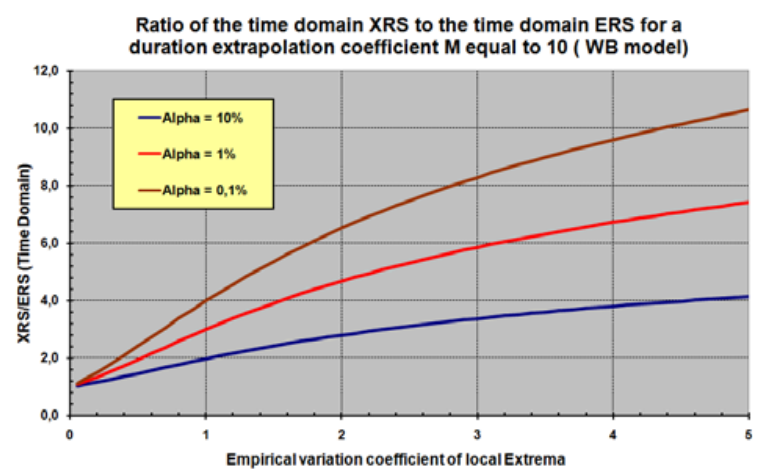

Figure 5. $\mathrm{XRS}_{\mathrm{tp}} / \mathrm{ERS}_{\mathrm{tp}}(\mathrm{WB}$ model) for $\mathrm{M}=10$.

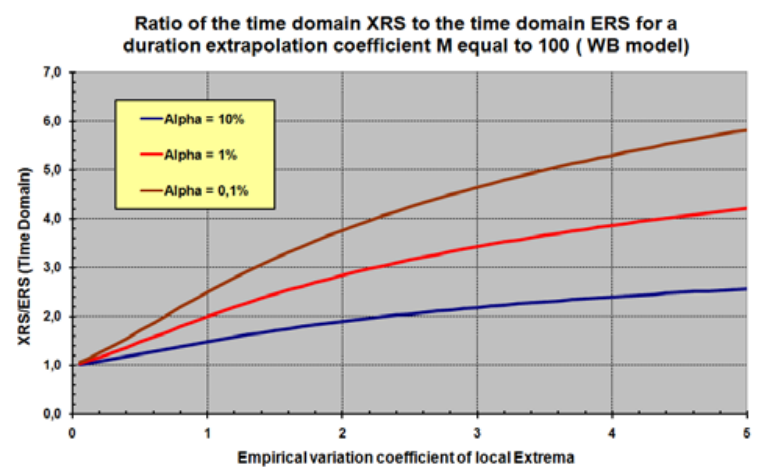

Figure 6. $\mathrm{XRS}_{\mathrm{tp}} / \mathrm{ERS}_{\mathrm{tp}}(\mathrm{WB}$ model) for $\mathrm{M}=100$.

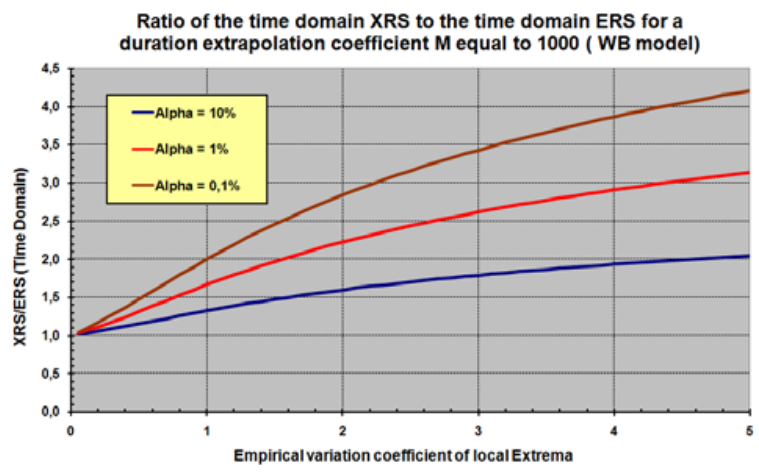

Figure 7. $\mathrm{XRS}_{\mathrm{tp}} / \mathrm{ERS}_{\mathrm{tp}}(\mathrm{WB}$ model) for $\mathrm{M}=1000$.

and Weibull models associated with time domain XRS calculations are similar, namely:

- the ratio between the time domain XRS and the time domain ERS increases with the coefficient of variation of local extremes of the stress process, for a given total load time,

- the ratio between the time domain XRS and the time domain ERS increases as the exceedance risk $\alpha$ reduces, for a given stress process (given coefficient of variation of local extremes and given load duration).

These time domain ERS and/or time domain XRS calculation approaches depend on the choice of the duration $T_{b}$ of the local extremes analysis block associated with the random non-Gaussian process considered. The scientific bases developed above show that it is then 
important to respect some fundamental criteria to define the optimum value of this analysis duration $\mathrm{T}_{\mathrm{b}}$ namely:

- minimise $\mathrm{T}_{\mathrm{b}}$, to obtain a sufficiently high number $\mathrm{N}$ [see relations (22) and (23)], so that the first two statistical moments of $Z_{\max }$ can be evaluated with good precision. Furthermore, this also tends to increase the duration extrapolation factor $M$ of the process [see relation (24)], thus justifying the use of the Gumbel asymptotic model.

- maximise $T_{b}$, to assure that the number of cycles is sufficient to evaluate the largest local maxima of the stress process, while assuring that extracted values are independent, block by block.

Since these two criteria are inherently antinomic, in practice the choice of the duration $\mathrm{T}_{\mathrm{b}}$ of the so-called "disjoint blocks" method is chosen as a function of the value of $T_{\text {ref }}$ and the required minimum value of $f_{0}$.

\section{Conclusions}

Response Spectra (RS) techniques, historically developed in terms of SRS for shock phenomena and spectral ERS for random vibration phenomena, cannot correctly characterise extreme responses of random non-Gaussian processes.

ERS time domain and XRS time domain techniques have been developed for this purpose and tested for Land Armament programs (VBCI and ARAVIS), highlighting their robustness and calculation speed characteristics.

These innovative time domain XRS calculation techniques have been recently proposed for AFNOR standardisation work, faced with the increase in the use of Response Spectra for the design of structures and for writing equipment qualification test specifications. Only the WB model was selected for standard NF X50-144-3, taking account of feedback acquired by Nexter Systems from the use of these two time domain XRS models proposed by the author [12].

As illustrated by this standard, these tools (time domain ERS and XRS) for characterisation of extreme values with imposed exceedance risk can very much broaden the application field of Response Spectrum calculation methods, and now cover the use of nonGaussian processes.
Since the non-Gaussian nature of processes is now taken into account by these stochastic time domain approaches, there is no longer any need to use deterministic time domain approaches presented in Sect. 3.2, particularly because they are not conservative.

\section{References}

[1] J.F. Imbert, Analyse des Structures par Eléments Finis-Cépadues Editions

[2] C. Lalanne, Vibrations et chocs mécaniques-Hermes Science publications, copyright Lavoisier, 1999 : Tome 5-Élaboration des spécifications

[3] Stephen H. Crandall and W.D Mark, Random vibration in mechanical systems (1963, Hardcover)

[4] B. Colin, La probabilisation des SRE : une réponse à la comparaison des sévérités d'environnement déterministe et stochastique, en termes de contraintes extrêmes, 25-27 Septembre 2007, ASTELAB 2007

[5] S.O Rice, Mathematical Analysis of Random Noise, B.S.T.J., 23, pp 282-332, 1944

[6] A. Preumont, Random Vibration and Spectral Analysis, Kluwer Academics Publishers, 1994

[7] B. Colin, «conception sécurisée des structures soumises aux valeurs extrêmes de processus stochastiques stationnaires», Mécanique \& Industries 10, 131-150 (2009), DOI: 10.151/meca/2009041

[8] J.S Bendat, Principles and Applications of Random Noise Theory, John Wiley \& Sons, 1958

[9] D.O. Smallwood, An improved recursive formula for calculating shock response spectra', The Shock and Vibration Bulletin, 51, Part 2, May 1981, p211-p217

[10] F.W. Cox, Efficient algorithms for calculating shock spectra on general purpose computers, The Shock and Vibration Bulletin, 53, Part 1, May 1983, p143p161

[11] NORMDEF 0101, Guide d'application de la démarche de personnalisation en environnement mécanique, DGA 2009, Edition 01, juillet 2009

[12] AFNOR, Norme NF X50-144-3, Démonstration de la tenue aux environnements-Conception et réalisation des essais en environnement-Partie 3 : Application de la démarche de personnalisation en environnement mécanique, 2014 